\title{
Read Only Memory Device
}

National Cancer Institute

\section{Source}

National Cancer Institute. Read Only Memory Device. NCI Thesaurus. Code C50048.

A type of data storage device which is manufactured with fixed contents. ROM is inherently non-volatile storage, retaining its contents even when the power is switched off. 